\title{
Analysis of an affine version of the Heston-Hull-White option pricing partial differential equation
}

\author{
Shimin Guo ${ }^{\mathrm{a}, \mathrm{b}, *}$, Lech A. Grzelak ${ }^{\mathrm{b}, \mathrm{c}}$, Cornelis W. Oosterlee ${ }^{\mathrm{b}, \mathrm{d}}$ \\ a School of Mathematics and Statistics, Xi'an Jiaotong University, 710049 Xi'an, China \\ ${ }^{\mathrm{b}} \mathrm{CWI}$ - Centrum Wiskunde \& Informatica, 1098XG Amsterdam, The Netherlands \\ ${ }^{c}$ Rabobank International, Utrecht, The Netherlands \\ d Delft Institute of Applied Mathematics, Delft University of Technology, Delft, The Netherlands
}

\section{A R T I C L E I N F O}

\section{Article history:}

Received 30 August 2012

Received in revised form 21 May 2013

Accepted 23 June 2013

Available online 28 June 2013

\section{Keywords:}

Heston-Hull-White model

cos method

ADI scheme

Error analysis

\begin{abstract}
A B S T R A C T
For European plain vanilla options, we investigate the difference between solutions obtained by the full-scale and an approximate Heston-Hull-White (HHW) model. Based on the corresponding two option pricing PDEs, we analyze the quality of the approximation. To confirm the accuracy of the analysis, we solve the HHW PDE, its approximating PDE as well as the PDE for the error, numerically, via a semi-discretization in space by a finite difference scheme on nonuniform spatial grids, and the Alternating Direction Implicit (ADI) scheme in time direction. Test cases with different parameter settings are considered. The effect of the financial parameters on the errors is discussed in detail.
\end{abstract}

(C) 2013 IMACS. Published by Elsevier B.V. All rights reserved.

\section{Introduction}

Calibration of asset price models in finance is a relevant and intensive task. The open parameters in stochastic differential equations (SDEs) governing the asset dynamics should be fitted in such a way that mathematically modeled and market observed option prices match possibly well. This task is typically done on the basis of so-called plain-vanilla European options, as these options include the market notion of the uncertainty in the future performance of assets, and their market prices are liquidly available. Many option prices have to be determined within the process of iteratively fitting model with market prices. Within the calibration process, highly efficient pricing of European options is thus mandatory.

Ideally an (semi-)analytic option price is available for the many calculations of the European option prices, but this is only the case for the most basic asset price dynamics, like the Black-Scholes dynamics. Alternatively, accurate approximate option values (or implied volatilities) by means of an asymptotic expansion can be used, see, for example, [7,13,17], but also here the (parameter) range of applicability may still be limited. A class of highly efficient numerical pricing techniques, pricing multiple options under different asset price dynamics, is the class of Fourier-based numerical integration. An exponent of this class of techniques is the COS method [6], which is based on Fourier cosine expansions, and on the availability of the characteristic function of the asset price dynamics. A characteristic function is easily derived, for example, for asset price dynamics in the class of affine jump diffusions [2], but characteristic functions have also been derived for local volatility models, based on approximations and asymptotic expansions, see [17].

Asset price models have become increasingly accurate in representing certain important features observed in market asset price dynamics. One of these features is the implied volatility smile or skew, which is a reflection of the fact that the market volatilities for options at different strike prices, for a single expiration date, are not constant. A smile can be

\footnotetext{
* Corresponding author at: School of Mathematics and Statistics, Xi'an Jiaotong University, 710049, Xi'an, China.

E-mail addresses: gsm861@126.com (S. Guo), Lech.Grzelak@rabobank.com (L.A. Grzelak), C.W.Oosterlee@cwi.nl (C.W. Oosterlee).
} 
observed in several different markets (equity, interest rate, FX, inflation and others). Stochastic volatility based asset price processes, the focus of the present paper, are able to mathematically model this property accurately.

For products with a longer time to the expiration date, it also makes sense to adopt a mathematical model in which the interest rate is modeled by a stochastic process, as in $[9,11]$. If we deal with financial products that are also sensitive to the equity volatility smile, we end up with a system of SDEs, in which the asset price, the volatility and the interest rate are state variables. We will consider here the Heston-Hull-White model, which may generate a smile pattern in the equity implied volatility structure, but not in interest rates, and as such it is an accurate model for financial products that are not sensitive to a skew pattern in the interest rates.

The resulting system of SDEs, with correlation coefficients connecting all processes, will generally not be an affine process.

Option pricing is often done with the discounted expected payoff approach, which is nothing but a Green's function integral which needs to be computed. The connection between the solution of the option pricing PDEs and the discounted expected payoff lies in the Feynman-Kac theorem. In many cases in option pricing, we do not have the Green's function (read, the conditional probability density function for the asset prices) available, but we do have its Fourier transform. This Fourier transform is called the characteristic function, and the theory by Duffie, Pan and Singleton [2] shows that for affine PDEs, i.e. PDEs with linear functions of the state variables in front of their partial derivatives, the characteristic function can easily be determined. This derivation technique is related to classical theory regarding the use of the Laplace transform for linear PDEs.

Unfortunately, for the full-scale Heston-Hull-White model, with a full matrix of correlations, a characteristic function is not easily available, as the resulting pricing PDE is not of the affine form. In [10], however, an approximation to the full-scale Heston-Hull-White model was derived, which belonged to the class of affine diffusions, and for which thus a characteristic function could be derived. Calibration of the full-scale HHW asset model has then been performed by the approximate model, and numerically it was observed in [10] that the difference between option prices computed by the approximate HHW model (highly efficiently by the COS method [6]) and the full-scale HHW model (by means of Monte Carlo simulation) was negligible small. However, these were mainly numerical experiments.

In the present paper we aim for a better theoretical understanding of the difference between the full-scale and the approximate HHW models. We consider the corresponding option pricing PDEs, and apply classical PDE error analysis to examine the quality of the approximation. In the case of the Heston-Hull-White model, the option pricing PDE will be threedimensional and an analytic option value is not available. We change measures, from the spot measure to the $T$-forward measure, so that the pricing PDE reduces to a two-dimensional PDE to facilitate the analysis. A numerical treatment of the two-dimensional PDE to confirm our error analysis is done by means of grid stretching and the ADI discretization, as in $[14,12]$. Elements from probability theory, as well as PDE theory and numerical analysis, are employed here.

\section{Problem description}

We start with the derivations that lead to the option pricing PDE under the Heston-Hull-White (HHW) asset dynamics. The HHW stochastic asset price process is governed by a stochastic interest rate, and a stochastic volatility. For financial derivative products with a long time to expiration this model can be employed.

We assume a complete probability space $(\Omega, \mathcal{F}, \mathbb{Q})$ and finite time horizon $[0, T] . \Omega$ is the set of all possible realizations of the stochastic economy between 0 and $T$. The information structure in this economy is represented by an augmented filtration $\mathcal{F}_{t}: t \in[0, T]$ with $\mathcal{F}_{T}$ the sigma algebra of distinguishable events at time $T$, and $\mathbb{Q}$ is the risk-neutral probability measure on elements of $\mathcal{F}$.

The model is defined, under the risk-neutral measure $\mathbb{Q}$, by the following system of SDEs:

$$
\begin{cases}\mathrm{d} S(t) / S(t)=r(t) \mathrm{d} t+\sqrt{v(t)} \mathrm{d} W_{x}^{\mathbb{Q}}(t), & S(0)>0, \\ \mathrm{~d} v(t)=\kappa(\bar{v}-v(t)) \mathrm{d} t+\gamma \sqrt{v(t)} \mathrm{d} W_{v}^{\mathbb{Q}}(t), & v(0)>0, \\ \mathrm{~d} r(t)=\lambda(\theta(t)-r(t)) \mathrm{d} t+\eta \mathrm{d} W_{r}^{\mathbb{Q}}(t), & r(0)>0 .\end{cases}
$$

Here, $S(t), v(t)$ and $r(t)(t \geqslant 0)$ are the stock price process, instantaneous volatility and short-term rate of interest, respectively. $\mathrm{d} W_{x}^{\mathbb{Q}}(t), \mathrm{d} W_{v}^{\mathbb{Q}}(t)$ and $\mathrm{d} W_{r}^{\mathbb{Q}}(t)$ are three correlated standard Brownian motions, with the correlation structure here given by:

$$
\left\{\begin{array}{l}
\mathrm{d} W_{x}(t) \mathrm{d} W_{v}(t)=\rho_{x, v} \mathrm{~d} t \\
\mathrm{~d} W_{x}(t) \mathrm{d} W_{r}(t)=\rho_{x, r} \mathrm{~d} t, \\
\mathrm{~d} W_{v}(t) \mathrm{d} W_{r}(t)=0 .
\end{array}\right.
$$

The parameters $\kappa$ and $\lambda$ control the speed of mean reversion of the volatility and the interest rate, respectively, $\eta$ represents the interest rate volatility, $\gamma$ the volatility of the $v(t)$-process, $\bar{v}$ and $\theta(t)$ are the long-run mean of the volatility and the interest rate process, respectively. Without loss of generality, we assume here that the correlation between the volatility and the interest rate process is equal to zero. If $2 \kappa \bar{v}>\gamma^{2}$, we refer to this condition as the Feller condition (being satisfied). The square-root process for the variance in (2.1) precludes negative values for $v(t)$, and if $v(t)$ reaches zero it can subsequently 
become positive. The Feller condition guarantees that $v(t)$ stays positive; otherwise, it may reach zero. The Feller condition is difficult to satisfy in practice. One often finds $2 \kappa \bar{v} \ll \gamma^{2}$ from market data, in which case the left tail of the variance density grows fast in value. This has, however, no impact on the pricing methods when dealing with European options.

Our interest is to price, under the risk-free measure $\mathbb{Q}$ associated to the bank account as numéraire, a European equity option, $V(t, S, v, r)$, defined as:

$$
V(t, S, v, r)=B(t) \mathbb{E}^{\mathbb{Q}}\left(\frac{1}{B(T)} H(T, S) \mid \mathcal{F}_{t}\right),
$$

where $H(T, S)$ represents a contingent claim (because we focus on efficient calibration, function $H(T, S)$ is assumed to be a basic put or a call option),

$$
H(T, S)= \begin{cases}\max (S-K, 0), & \text { for a call option, } \\ \max (K-S, 0), & \text { for a put option. }\end{cases}
$$

Further, $B(t)$ is the money-savings account, given by $B(t)=\exp \left(\int_{0}^{t} r(s) \mathrm{d} s\right), T$ is the expiration date of the option, and the aim is to determine the value of the option, for example, at $t=t_{0}=0$.

By the non-arbitrage assumptions, one can derive the corresponding HHW PDE which defines the value of a Europeanstyle option:

$$
\begin{aligned}
0= & \frac{\partial V}{\partial t}+r S \frac{\partial V}{\partial S}+\kappa(\bar{v}-v) \frac{\partial V}{\partial v}+\lambda(\theta(t)-r) \frac{\partial V}{\partial r}+\frac{1}{2} v S^{2} \frac{\partial^{2} V}{\partial S^{2}}+\frac{1}{2} \gamma^{2} v \frac{\partial^{2} V}{\partial v^{2}}+\frac{1}{2} \eta^{2} \frac{\partial^{2} V}{\partial r^{2}} \\
& +\rho_{x, v} \gamma v S \frac{\partial^{2} V}{\partial S \partial v}+\rho_{x, r} \eta \sqrt{v} S \frac{\partial^{2} V}{\partial S \partial r}-r V,
\end{aligned}
$$

with $t \in[0, T)$, and spatial coordinates $\{S, v, r\} \in[0,+\infty) \times[0,+\infty) \times(-\infty,+\infty)$. Of course, we will solve the discrete version of the PDE on a finite domain.

As the final condition we have $V(T, S, v, r)=H(T, S)$.

Boundary conditions at $S=0$ are essentially the same ones as used when dealing with the well-known Black-Scholes equation, i.e., for a call option

$$
V(t, S=0, v, r)=0 .
$$

We prescribe the boundary condition at $v=0$ for PDE (2.5) by substituting $v=0$ in the original PDE. This boundary condition has been examined in this context in [4]. Furthermore,

$$
\frac{\partial V(t, S, v, r)}{\partial v}=0
$$

for $v \rightarrow+\infty$. For extreme values of the spot interest rate, we use

$$
\frac{\partial V(t, S, v, r)}{\partial r}=0
$$

for the boundary condition at $r \rightarrow \pm \infty$. See [12] for details. PDE (2.5) is three-dimensional and it is nontrivial to solve it analytically.

It is however well known that a second-order linear PDE with constants or linear functions of independent variables in front of the derivatives can be solved highly efficiently by means of, for example, the Laplace transform. We call linear PDEs with linear functions of the independent variables in front of their derivatives affine PDEs, as in [2]. Affine PDEs originate from the application of Itô's lemma to a system of (asset pricing) SDEs from the class of affine jump diffusions. For this class of stochastic models we can derive the characteristic function, which is the Fourier transform of the conditional probability density function. By means of the Feynman-Kac theorem we can solve affine PDEs by computation of a discounted expected payoff.

The HHW stochastic system is not in the class of affine diffusions, because not all the terms in front of the derivatives in PDE (2.5) are of the affine form. Therefore, we cannot use the standard techniques from [2] to derive the corresponding characteristic function. Clearly the problem would be solved by setting the correlation between the stock and the interest rate to zero, however, a nonzero correlation between these processes is of main interest.

\subsection{The HHW model under the T-forward measure}

In order to reduce the complexity, it is convenient to switch between two pricing measures, i.e. we focus on the forward stock price, $F(t)$, defined by:

$$
F(t)=\frac{S(t)}{P(t, T)},
$$


with $P(t, T)$ the zero-coupon bond (ZCB), expiring at time $T$, which pays 1 unit of currency at time $T$. By switching from the domestic, risk-neutral measure, $\mathbb{Q}$, to the $T$-forward measure, $\mathbb{Q}^{T}$, discounting will be decoupled ${ }^{1}$ from the expectation operator, i.e.,

$$
V(t, S, v)=P(t, T) \mathbb{E}^{T}\left(\max (F(T)-K, 0) \mid \mathcal{F}_{t}\right),
$$

where independent variable $r$ does not appear as an argument anymore. In order to determine the dynamics of the forward $F(t)$ in (2.6), we apply Itô's formula,

$$
\mathrm{d} F(t)=\frac{1}{P(t, T)} \mathrm{d} S(t)-\frac{S(t)}{P^{2}(t, T)} \mathrm{d} P(t, T) .
$$

The process for the forward stock, $F(t)$, is a martingale under the $T$-forward measure and therefore we won't encounter any dt-terms in its dynamics (2.8), for details see [19]. The SDE of $F(t)$ will consist only of the volatility terms of $S(t)$ and $P(t, T)$. In expression (2.8) the dynamics for $P(t, T)$ are present. Since the short rate process is driven here by the Hull-White model, the dynamics for the ZCB are given by:

$$
\frac{\mathrm{d} P(t, T)}{P(t, T)}=r(t) \mathrm{d} t+\eta B_{r}(t, T) \mathrm{d} W_{r}^{\mathbb{Q}}(t),
$$

where $B_{r}(t, T):=\frac{1}{\lambda}\left(\mathrm{e}^{-\lambda(T-t)}-1\right)$. By combining Eqs. (2.6), (2.8) and (2.9), we find:

$$
\left\{\begin{array}{l}
\mathrm{d} F(t) / F(t)=\sqrt{v(t)} \mathrm{d} W_{F}^{T}-\eta B_{r}(t, T) \mathrm{d} W_{r}^{T}, \\
\mathrm{~d} v(t)=\kappa(\bar{v}-v(t)) \mathrm{d} t+\gamma \sqrt{v(t)} \mathrm{d} W_{v}^{T}(t),
\end{array}\right.
$$

with $\mathrm{d} W_{F}^{T}(t) \mathrm{d} W_{v}^{T}(t)=\rho_{F, v} \mathrm{~d} t$ and $\mathrm{d} W_{F}^{T}(t) \mathrm{d} W_{r}^{T}(t)=\rho_{F, r} \mathrm{~d} t$.

Based on non-arbitrage arguments the following Fokker-Planck forward PDE can be derived:

$$
\begin{aligned}
-\frac{\partial V}{\partial t}= & \kappa(\bar{v}-v) \frac{\partial V}{\partial v}+\frac{1}{2} \gamma^{2} v \frac{\partial^{2} V}{\partial v^{2}}+F^{2}\left(\frac{1}{2} v+\frac{1}{2} \eta^{2} B_{r}^{2}(t, T)-\rho_{F, r} \eta B_{r}(t, T) \sqrt{v}\right) \frac{\partial^{2} V}{\partial F^{2}} \\
& +\rho_{F, v} \gamma F v \frac{\partial^{2} V}{\partial F \partial v} .
\end{aligned}
$$

Also in PDE formulation (2.11) not all terms are of the affine form, as it contains the non-affine factor $\rho_{F, r} \eta B_{r}(t, T) \sqrt{v}$, with a nonzero correlation. The same is true after making a log-transformation, which is the common transformation to reach an affine system of SDEs.

Still we cannot use standard techniques from [2] to find the corresponding characteristic function.

We therefore define an approximation to PDE (2.11) for a highly efficient computation of an approximate solution. A possibility to approximate the PDE by an affine PDE is to replace the non-affine term $\sqrt{v}$ in (2.11) by a time-dependent function, $\Phi(t, v(0))$. In detail, we project $\sqrt{v(t)}$ onto its expectation, $\mathbb{E}^{T}\left(\sqrt{v(t)} \mid \mathcal{F}_{0}\right)$, which gives us the following, approximating, pricing PDE, for which we denote the solution by $\widetilde{V}$,

$$
\begin{aligned}
-\frac{\partial \widetilde{V}}{\partial t}= & \kappa(\bar{v}-v) \frac{\partial \widetilde{V}}{\partial v}+\frac{1}{2} \gamma^{2} v \frac{\partial^{2} \widetilde{V}}{\partial v^{2}}+F^{2}\left(\frac{1}{2} v+\frac{1}{2} \eta^{2} B_{r}^{2}(t, T)-\rho_{F, r} \eta B_{r}(t, T) \Phi(t, v(0))\right) \frac{\partial^{2} \widetilde{V}}{\partial F^{2}} \\
& +\rho_{F, v} \gamma v F \frac{\partial^{2} \widetilde{V}}{\partial F \partial v},
\end{aligned}
$$

with the same boundary and final conditions as (2.11).

We will analyze the following two closed-form expressions for the expectation of the square-root process, $\Phi(t, v(0))=$ $\mathbb{E}^{T}\left(\sqrt{v(t)} \mid \mathcal{F}_{0}\right)$

1. By [3] and simplifications as in [15], a closed-form expression for $\mathbb{E}^{T}\left(\sqrt{v(t)} \mid \mathcal{F}_{0}\right)$ can be determined, see [10]:

$$
\Phi(t, v(0)) \equiv \mathbb{E}^{T}\left(\sqrt{v(t)} \mid \mathcal{F}_{0}\right)=\sqrt{2 c(t)} \mathrm{e}^{-\omega(t) / 2} \sum_{k=0}^{\infty} \frac{1}{k !}(\omega(t) / 2)^{k} \frac{\Gamma\left(\frac{1+d}{2}+k\right)}{\Gamma\left(\frac{d}{2}+k\right)}
$$

with

$$
c(t)=\frac{1}{4 \kappa} \gamma^{2}\left(1-\mathrm{e}^{-\kappa t}\right), \quad d=\frac{4 \kappa \bar{v}}{\gamma^{2}}, \quad \omega(t)=\frac{4 \kappa v(0) \mathrm{e}^{-\kappa t}}{\gamma^{2}\left(1-\mathrm{e}^{-\kappa t}\right)},
$$

and Gamma function $\Gamma(z)=\int_{0}^{\infty} t^{z-1} \mathrm{e}^{-t} \mathrm{~d} t$. Note that $t=0$ is a singular point for this function.

\footnotetext{
1 Which is different from formulation (2.3).
} 
2. The expectation for the square root process in (2.13) is not of a trivial form, and therefore we propose a simple approximation as well.

A simplified closed-form approximation for $\mathbb{E}^{T}\left(\sqrt{v(t)} \mid \mathcal{F}_{0}\right)$ is given by, see [10],

$$
\Phi(t, v(0)) \equiv \mathbb{E}^{T}\left(\sqrt{v(t)} \mid \mathcal{F}_{0}\right) \approx \alpha+\beta \mathrm{e}^{-\xi t},
$$

with

$$
\begin{aligned}
& \alpha=\sqrt{\bar{v}-\frac{\gamma^{2}}{8 \kappa}, \quad \beta=\sqrt{v(0)}-\alpha, \quad \xi=-\log \left(\beta^{-1}(\Lambda(1)-\alpha)\right),} \\
& \Lambda(t)=\sqrt{c(t)(\omega(t)-1)+d c(t)+\frac{d c(t)}{2(d+\omega(t))}},
\end{aligned}
$$

where $c(t), d$ and $\omega(t)$ are as presented in Eq. (2.14).

\subsection{The Fourier Cosine Method (COS) for European options}

With a deterministic function $\Phi(t, v(0))$ the approximate pricing PDE under the log transform is of the affine form, so that highly efficient pricing techniques can be applied to solve the PDE. We can determine the characteristic function of an affine process. With the characteristic function available one can apply pricing techniques within the discounted expected payoff formulation. The density and its characteristic function, $f(y \mid x)$ and $\varphi(u, x)$ form a Fourier pair,

$$
\varphi(u, x)=\int_{\mathbb{R}} e^{i y u} f(y \mid x) \mathrm{d} y, \quad f(y \mid x)=\frac{1}{2 \pi} \int_{\mathbb{R}} \mathrm{e}^{-i u y} \varphi(u, x) \mathrm{d} u,
$$

which are the forward and inverse Fourier integrals, respectively.

The Fourier cosine pricing method, named the $\cos$ method [6], is based on the risk-neutral option valuation formula (discounted expected payoff approach):

$$
V\left(t_{0}, x, v\right)=e^{-r \Delta t} \int_{-\infty}^{\infty} V(T, y, v) f(y \mid x) \mathrm{d} y,
$$

where $V\left(t_{0}, x, v\right)$ is the present option value, as in (2.7), $r$ the interest rate, $\Delta t=T-t_{0}$ and $x, y$ can be any monotone function of the underlying asset at initial time $t_{0}$ and the expiration date $T$. Function $V(T, y, v)$, which equals the payoff function, is known, but the conditional density function, $f(y \mid x)$ in (2.18), typically is not.

This integral formulation resembles the Green's function integral. The characteristic function serves as the Fourier transform of the Green's function, so that it makes sense to solve affine PDEs in the Fourier domain. This can be done highly efficiently by means of the Fast Fourier Transform or by means of Fourier expansions. The availability of the characteristic function can be interpreted as follows: For many affine diffusion processes, we do not have an explicit formulation of the Green's function available, but the Fourier transform of the Green's function, i.e. the characteristic function, is known.

We approximate the conditional density function on a truncated domain, by a truncated Fourier cosine expansion, which recovers the conditional density function from its characteristic function (see [6]) as follows:

$$
f(y \mid x) \approx \frac{2}{b-a} \sum_{k=0}^{N-1} \operatorname{Re}\left(\varphi\left(\frac{k \pi}{b-a}, x\right) \exp \left(-i \frac{a k \pi}{b-a}\right)\right) \cos \left(k \pi \frac{y-a}{b-a}\right),
$$

with $\varphi(u, x)$ the characteristic function of $f(y \mid x) ; a, b$ determine a truncated domain and Re means taking the real part of the argument. The prime at the sum symbol indicates that the first term in the expansion is multiplied by one-half. The size of the truncated domain can be determined with the help of the cumulants [6]. ${ }^{2}$

Replacing $f(y \mid x)$ by its approximation (2.19) in Eq. (2.18) and interchanging integration and summation gives the COS formula for computing the values of European options:

$$
V\left(t_{0}, x, v\right)=\mathrm{e}^{-r \Delta t} \sum_{k=0}^{N-1} \operatorname{Re}\left(\varphi\left(\frac{k \pi}{b-a}, x\right) \mathrm{e}^{-i k \pi \frac{a}{b-a}}\right) P_{k}
$$

\footnotetext{
2 For example so that $\left|\int_{\mathrm{R}} f(y \mid x) \mathrm{d} y-\int_{a}^{b} f(y \mid x) \mathrm{d} y\right|<T O L$.
} 
where:

$$
P_{k}=\frac{2}{b-a} \int_{a}^{b} V(T, y, v) \cos \left(k \pi \frac{y-a}{b-a}\right) \mathrm{d} y,
$$

are the Fourier cosine coefficients of $V(T, y, v)$, that are available in closed form for several payoff functions, like for plain vanilla puts and calls, but also for example for discontinuous payoffs like for digital options.

It was found by analysis in [6], that, with integration interval $[a, b]$ chosen sufficiently wide, the series truncation error dominates the overall error. For conditional density functions $f(y \mid x) \in C^{\infty}((a, b) \subset \mathbb{R})$, the method converges exponentially. Option pricing by means of formula $(2.20)$ is highly efficient. This way PDE (2.12) can be solved in milliseconds.

\subsection{Properties of covariance matrix}

A proper numerical treatment of PDEs (2.11) or (2.12) requires that the corresponding instantaneous covariance matrix should be positive definite.

It is well known that a matrix $A$ is positive definite when all its sub-matrices have a positive determinant, known as Sylvester's criterion.

Since we deal with a $2 \times 2$ covariance matrix, after the change to the $T$-forward measure, one determinant needs to be positive $\operatorname{det}(\mathbf{A})>0$, next to the upper left matrix element, i.e. for (2.11),

$$
\mathbf{A}=\left[\begin{array}{cc}
F^{2}(t)\left(\frac{1}{2} v(t)+\frac{1}{2} \eta^{2} B_{r}^{2}(t, T)-\rho_{F, r} \eta B_{r}(t, T) \sqrt{v(t)}\right) & \frac{1}{2} \rho_{F, v} \gamma v(t) F(t) \\
\frac{1}{2} \rho_{F, v} \gamma v(t) F(t) & \frac{1}{2} \gamma^{2} v(t)
\end{array}\right] .
$$

By basic manipulations, we find $\operatorname{det}(\mathbf{A})>0 \Leftrightarrow$

$$
\frac{1}{2} \gamma^{2} v(t) F^{2}(t)\left(\frac{1}{2} v(t)+\frac{1}{2} \eta^{2} B_{r}^{2}(t, T)-\rho_{F, r} \eta B_{r}(t, T) \sqrt{v(t)}\right)-\frac{1}{4} \rho_{F, v}^{2} \gamma^{2} v^{2}(t) F^{2}(t)>0
$$

Dividing both sides by $\gamma^{2} v(t) F^{2}(t)$ gives us

$$
\frac{1}{4} v(t)-\chi(t, T)-\frac{1}{4} \rho_{F, v}^{2} v(t)>0
$$

where $\chi(t, T)=-\left(\frac{1}{4} \eta^{2} B_{r}^{2}(t, T)-\frac{1}{2} \rho_{F, r} \eta B_{r}(t, T) \sqrt{v(t)}\right)$. Since $v(t)>0$ and $B_{r}(t, T)<0$, it is easy to see that $\chi(t, T)$ is always non-positive.

Finally, we find:

$$
\frac{1}{4} v(t)\left(1-\rho_{F, v}^{2}\right)>\chi(t, T)
$$

Clearly, the left-hand side of the above inequality is positive for $\left|\rho_{F, v}\right|<1$ and $v(t)>0$, whereas $\chi(t, T)$ is always nonpositive for the conditions described before.

By the same procedure as above, we can get the following condition for the approximate HHW PDE (2.12),

$$
\frac{1}{4} v(t)\left(1-\rho_{F, v}^{2}\right)>\Lambda(t, T)
$$

where $\Lambda(t, T)=-\left(\frac{1}{4} \eta^{2} B_{r}^{2}(t, T)-\frac{1}{2} \rho_{F, r} \eta B_{r}(t, T) \Phi(t, v(0))\right)$. Since $\Phi(t, v(0))=\mathbb{E}^{T}\left(\sqrt{v(t)} \mid \mathcal{F}_{0}\right)>0, \Lambda(t, T)$ is always nonpositive. Therefore, we obtain a similar result as for the full-scale HHW PDE (2.11), which means that the approximations made do not pose any difficulty regarding the properties of the covariance matrix.

\section{Error analysis}

We now perform error analysis to gain insight between the differences in the solutions $V$ and $\widetilde{V}$ of respectively the full-scale (2.11) and approximate HHW PDE (2.12). We start with a heuristic qualitative statement.

\subsection{Error analysis - stochastic approach}

The factors multiplying the second-order partial derivatives in the pricing PDE correspond to the terms from the instantaneous covariance matrix of the underlying stochastic model. 
For the $\log$-forward, $x(t)=\log F(t)$, with variance process $v(t)$ as in (2.11), the corresponding option pricing PDE reads:

$$
\begin{aligned}
-\frac{\partial V}{\partial t}= & \kappa(\bar{v}-v) \frac{\partial V}{\partial v}+\frac{1}{2} \gamma^{2} v \frac{\partial^{2} V}{\partial v^{2}}+\left(\frac{1}{2} v+\frac{1}{2} \eta^{2} B_{r}^{2}(t, T)-\rho_{F, r} \eta B_{r}(t, T) \sqrt{v}\right)\left(\frac{\partial^{2} V}{\partial x^{2}}-\frac{\partial V}{\partial x}\right) \\
& +\rho_{F, v} \gamma v \frac{\partial^{2} V}{\partial x \partial v} .
\end{aligned}
$$

The associated instantaneous covariance matrix for state vector $\mathbf{X}(t)=[x(t), v(t)]^{\mathrm{T}}$ is thus given by:

$$
\mathrm{d} \mathbf{X}(t) \mathrm{d} \mathbf{X}(t)^{\mathrm{T}}=\left[\begin{array}{cc}
v(t)+\eta^{2} B_{r}^{2}(t, T)-2 \rho_{F, r} \eta B_{r}(t, T) \sqrt{v(t)} & \rho_{F, v} \gamma v(t) \\
\rho_{F, v} \gamma v(t) & \gamma^{2} v(t)
\end{array}\right] .
$$

With the projection $\sqrt{v(t)} \approx \mathbb{E}^{T}\left(\sqrt{v(t)} \mid \mathcal{F}_{0}\right)$ we need to analyze the error due to this approximation. If the variance of the square-root process, $\operatorname{Var}\left(\sqrt{v(t)} \mid \mathcal{F}_{0}\right)$ equals zero then obviously $\sqrt{v(t)} \equiv \mathbb{E}^{T}\left(\sqrt{v(t)} \mid \mathcal{F}_{0}\right)$.

Variance $\operatorname{Var}\left(\sqrt{v(t)} \mid \mathcal{F}_{0}\right)$ is given by:

$$
\begin{aligned}
\operatorname{Var}\left(\sqrt{v(t)} \mid \mathcal{F}_{0}\right) & =\mathbb{E}^{T}\left(v(t) \mid \mathcal{F}_{0}\right)-\left(\mathbb{E}^{T}\left(\sqrt{v(t)} \mid \mathcal{F}_{0}\right)\right)^{2} \\
& =c(t)(d+\omega(t))-\left(\mathbb{E}^{T}\left(\sqrt{v(t)} \mid \mathcal{F}_{0}\right)\right)^{2},
\end{aligned}
$$

where $c(t), d$ and $\omega(t)$ are given in (2.14). Since the second moment in Eq. (3.3) is always positive, bounds for variance $\operatorname{Var}\left(\sqrt{v(t)} \mid \mathcal{F}_{0}\right)$ are given by:

$$
0 \leqslant \operatorname{Var}\left(\sqrt{v(t)} \mid \mathcal{F}_{0}\right) \leqslant c(t)(d+\omega(t))=\bar{v}-\bar{v} \mathrm{e}^{-\kappa t}+v(0) \mathrm{e}^{-\kappa t} .
$$

The upper bound for the variance is thus based on the values of $v(0), \bar{v}$ and mean reversion parameter $\kappa$.

When we consider $\bar{v} \equiv v(0)$ (which is often the case in practice) the upper bound for the variance equals $\bar{v}$, so that for small values of $\bar{v}$ higher accuracy is expected.

In the limit $t \rightarrow \infty$, we have, by [3], that:

$$
\mathbb{E}^{T}\left(\sqrt{v(t)} \mid \mathcal{F}_{0}\right)=\sqrt{2 c(t)} \frac{\Gamma\left(\frac{1+d}{2}\right)}{\Gamma\left(\frac{d}{2}\right)}{ }_{1} F_{1}\left(-\frac{1}{2}, \frac{d}{2},-\frac{\omega(t)}{2}\right),
$$

where ${ }_{1} F_{1}(a ; b ; z)$ is a confluent hyper-geometric function. Since $\lim _{t \rightarrow \infty} \omega(t)=0$, and $\lim _{t \rightarrow \infty} c(t)=\gamma^{2} /(4 \kappa)$, the variance in the limit equals:

$$
\begin{aligned}
\lim _{t \rightarrow \infty} \operatorname{Var}(\sqrt{v(t)}) & =c(\infty)(d+\omega(\infty))-\left(\sqrt{2 c(\infty)} \frac{\Gamma\left(\frac{1+d}{2}\right)}{\Gamma\left(\frac{d}{2}\right)}{ }_{1} F_{1}\left(-\frac{1}{2}, \frac{d}{2},-\frac{\omega(\infty)}{2}\right)\right)^{2} \\
& =\bar{v}-\frac{\gamma^{2}}{2 \kappa}\left(\frac{\Gamma\left(\frac{1+d}{2}\right)}{\Gamma\left(\frac{d}{2}\right)}\right)^{2}
\end{aligned}
$$

With a closer look at the non-affine first element of the covariance matrix in (3.2), i.e. $v(t)+\eta^{2} B_{r}^{2}(t, T)-$ $2 \rho_{F, r} \eta B_{r}(t, T) \sqrt{v(t)}$, we find that, for $t \rightarrow T$, the term $B_{r}(t, T)$ converges to zero, and the last term, $2 \rho_{F, r} \eta B_{r}(t, T) \sqrt{v(t)}$, therefore contributes only for $0<t<T$. On the other hand, the variance of $\sqrt{v(t)}$ is smallest for small values of $t$, as for $t \rightarrow 0, \sqrt{v(t)} \rightarrow \sqrt{v(0)}$. These observations indicate that the projection of the non-affine term onto the expectation is expected to provide a highly satisfactory approximation.

\subsection{PDE error analysis}

In this section we turn to classical PDE error analysis, to also make some quantitative statements about the approximation errors encountered. The PDEs in (2.11) and (2.12) can be written, in shorthand notation, as follows:

$$
\left\{\begin{array}{l}
\frac{\partial V}{\partial t}+L_{1} V=0, \\
\frac{\partial \widetilde{V}}{\partial t}+L_{2} \widetilde{V}=0,
\end{array}\right.
$$

with the corresponding operators $L_{1}$, as in (2.11) and $L_{2}$, as in (2.12). Again, $V$ is the solution from the full-scale HHW PDE, whereas $\widetilde{V}$ is the solution from the approximating PDE (which can be determined highly efficiently by the COS method). Both PDEs are accompanied by the same boundary and final conditions. For the error, $e:=V-\widetilde{V}$, we find:

$$
\frac{\partial e}{\partial t}+L_{1} V-L_{2} \widetilde{V}=0
$$


which can be re-written as:

$$
\frac{\partial e}{\partial t}+L_{1} V-\left(L_{1} \tilde{V}+\left(L_{2}-L_{1}\right) \tilde{V}\right)=0
$$

and we arrive at the following equation:

$$
\frac{\partial e}{\partial t}+L_{1} e=\left(L_{2}-L_{1}\right) \tilde{V}
$$

subject to homogeneous boundary and final conditions. Notice that the right-hand side of the equation serves as a source term.

In fact, the following two PDEs can be used for error analysis purposes:

$$
\left\{\begin{array}{l}
\frac{\partial e}{\partial t}+L_{2} e=\left(L_{2}-L_{1}\right) V \\
\frac{\partial e}{\partial t}+L_{1} e=\left(L_{2}-L_{1}\right) \widetilde{V}
\end{array}\right.
$$

Remark 1 (Existence of a strong solution). It is easy to see that the variable-coefficient PDE operators governing (3.7) and (3.11) can be written in the form [5,8]:

$$
L u=\frac{1}{2}\left(c_{11} \frac{\partial^{2} u}{\partial F^{2}}+c_{12} \frac{\partial^{2} u}{\partial F \partial v}+c_{21} \frac{\partial^{2} u}{\partial v \partial F}+c_{22} \frac{\partial^{2} u}{\partial v^{2}}\right)+b \frac{\partial u}{\partial v}-\frac{\partial u}{\partial t},
$$

where $u=u(t, F, v)$ represents $V, \widetilde{V}$ and $e$ in Eqs. (3.7) and (3.11), respectively, $c_{i j}=c_{i j}(t, F, v)(i, j=1,2)$ and $b=b(t, F, v)$ are the corresponding variable coefficients. We suppose that the operator $L$ is uniformly parabolic, i.e. there exists a positive constant $\mu$ such that

$$
\mu^{-2}|\xi|^{2} \leqslant \sum_{i, j=1}^{2} c_{i j} \xi_{i} \xi_{j} \leqslant \mu^{2}|\xi|^{2}, \quad \xi \in \mathbb{R}^{2}
$$

Furthermore, from the expressions of the coefficients in Eqs. (2.11) and (2.12), we can see that these coefficients are bounded and Hölder continuous. Consider the problem

$$
\begin{cases}L u=f, & \text { in } S_{T}=[0, T] \times \mathbb{R}^{2}, \\ u(T, F, v)=\varphi, & \text { on } \mathbb{R}^{2} .\end{cases}
$$

Here, $L$ is the parabolic operator in Eq. (3.12),

$$
\varphi= \begin{cases}\max (F(T)-K, 0), & \text { for a call option, } \\ \max (K-F(T), 0), & \text { for a put option, }\end{cases}
$$

in Eq. (3.7), $\varphi=0$ in Eq. (3.11), and $f$ represents the second term in both equations (with $f=0$ in (3.7)). Clearly, for all cases, $\varphi$ is continuous on $\bar{S}_{T}$, locally Lipschitz continuous and for every bounded open set $0, \overline{0} \subseteq S_{T}$, there exists a constant $C$ such that

$$
\left(\xi_{11} \frac{\partial^{2} \varphi}{\partial F^{2}}+\xi_{12} \frac{\partial^{2} \varphi}{\partial F \partial v}+\xi_{21} \frac{\partial^{2} \varphi}{\partial v \partial F}+\xi_{22} \frac{\partial^{2} \varphi}{\partial v^{2}}\right) \geqslant C|\xi|^{2} \quad \text { in } 0, \xi \in \mathbb{R}^{2},
$$

in the sense of distributions

$$
\left(\xi_{11} \int_{O} \varphi \frac{\partial^{2} \psi}{\partial F^{2}}+\xi_{12} \int_{O} \varphi \frac{\partial^{2} \psi}{\partial F \partial v}+\xi_{21} \int_{O} \varphi \frac{\partial^{2} \psi}{\partial v \partial F}+\xi_{22} \int_{O} \varphi \frac{\partial^{2} \psi}{\partial v^{2}}\right) \geqslant C|\xi|^{2} \int_{O} \psi
$$

for all $\xi \in \mathbb{R}^{2}$ and $\psi \in C_{0}^{\infty}(0)$ with $\psi \geqslant 0$. Under suitable additional assumptions, the existence and uniqueness of solution can be obtained following the techniques in $[18,1]$.

Based on the form in (3.10), multiplying both sides by $e$, and integration over domain $\Omega$, gives us:

$$
\int_{\Omega} e \frac{\partial e}{\partial t} \mathrm{~d} \Omega+\int_{\Omega} e L_{1} e \mathrm{~d} \Omega=\int_{\Omega} e\left(L_{2}-L_{1}\right) \tilde{V} \mathrm{~d} \Omega .
$$

Integration by parts, as follows,

$$
\int_{\Omega} e \frac{\partial e}{\partial t} \mathrm{~d} \Omega=\frac{1}{2} \frac{\mathrm{d}}{\mathrm{d} t} \int_{\Omega} e^{2} \mathrm{~d} \Omega=\frac{1}{2} \frac{\mathrm{d}}{\mathrm{d} t}\|e\|_{L^{2}(\Omega)}^{2},
$$


inserted in Eq. (3.14), results in:

$$
\frac{1}{2} \frac{\mathrm{d}}{\mathrm{d} t}\|e\|_{L^{2}(\Omega)}^{2}=-\int_{\Omega} e L_{1} e \mathrm{~d} \Omega+\int_{\Omega} e\left(L_{2}-L_{1}\right) \tilde{V} \mathrm{~d} \Omega .
$$

The $V$-ellipticity of the form $\int_{\Omega} e L_{1} e \mathrm{~d} \Omega$ [16], following from the Lax-Friedrich (also known as the Poincaré) inequality, gives

$$
\int_{\Omega} e L_{1} e \mathrm{~d} \Omega \geqslant \alpha \int_{\Omega} e^{2} \mathrm{~d} \Omega=: \alpha\|e\|_{L^{2}(\Omega)}^{2},
$$

which implies unique solvability of the problem. Inserting this result in (3.16) results in:

$$
\frac{1}{2} \frac{\mathrm{d}}{\mathrm{d} t}\|e\|_{L^{2}(\Omega)}^{2} \leqslant-\alpha\|e\|_{L^{2}(\Omega)}^{2}+\int_{\Omega} e\left(L_{2}-L_{1}\right) \widetilde{V} \mathrm{~d} \Omega .
$$

We further have:

$$
\begin{aligned}
\frac{1}{2} \frac{\mathrm{d}}{\mathrm{d} t}\|e\|_{L^{2}(\Omega)}^{2}+\alpha\|e\|_{L^{2}(\Omega)}^{2} & \leqslant \int_{\Omega} e\left(L_{2}-L_{1}\right) \tilde{V} \mathrm{~d} \Omega \\
& \leqslant\|e\|_{L^{2}(\Omega)}\left\|\left(L_{2}-L_{1}\right) \widetilde{V}\right\|_{L^{2}(\Omega)},
\end{aligned}
$$

or,

$$
\frac{\mathrm{d}}{\mathrm{d} t}\|e\|_{L^{2}(\Omega)}+\alpha\|e\|_{L^{2}(\Omega)} \leqslant\left\|\left(L_{2}-L_{1}\right) \widetilde{V}\right\|_{L^{2}(\Omega)} .
$$

We then apply Grönwall's lemma, i.e.,

$$
\frac{\mathrm{d}}{\mathrm{d} t}\left(\mathrm{e}^{\alpha t}\|e\|_{L^{2}(\Omega)}\right) \leqslant\left\|\left(L_{2}-L_{1}\right) \widetilde{V}\right\| \mathrm{e}^{\alpha t} .
$$

Integrating both sides gives:

$$
\mathrm{e}^{\alpha t}\|e\|_{L^{2}(\Omega)}(t)-\|e\|_{L^{2}(\Omega)}(0) \leqslant \int_{0}^{t}\left\|\left(L_{2}-L_{1}\right) \widetilde{V}\right\|_{L^{2}(\Omega)} \mathrm{e}^{\alpha s} \mathrm{~d} s .
$$

Since the error $e$, at initial point $t=0$, vanishes, we have:

$$
\begin{aligned}
\|e\|_{L^{2}(\Omega)} & \leqslant \int_{0}^{t}\left\|\left(L_{2}-L_{1}\right) \widetilde{V}\right\|_{L^{2}(\Omega)} \mathrm{e}^{\alpha(s-t)} \mathrm{d} s \\
& \leqslant \frac{1}{\alpha}\left(1-\mathrm{e}^{-\alpha t}\right) \sup _{s \in(0, t)}\left\|\left(L_{2}-L_{1}\right) \widetilde{V}\right\|_{L^{2}(\Omega)} \\
& \leqslant \frac{1}{\alpha} \sup _{s \in(0, t)}\left\|\left(L_{2}-L_{1}\right) \widetilde{V}\right\|_{L^{2}(\Omega)^{\prime}} .
\end{aligned}
$$

We focus on the norm $\left\|\left(L_{2}-L_{1}\right) \widetilde{V}\right\|_{L^{2}(\Omega)}$, and find the following perturbation of the operator:

$$
\left(L_{2}-L_{1}\right) \widetilde{V}=F^{2} \rho_{F, r} \eta B_{r}(t, T)(\Phi(t, v(0))-\sqrt{v}) \frac{\partial^{2} \widetilde{V}}{\partial F^{2}} .
$$

We can asses the appropriate norm:

$$
\begin{aligned}
\left\|\left(L_{2}-L_{1}\right) \tilde{V}\right\|_{L^{2}(\Omega)} & =\left|\rho_{F, r} \eta B_{r}(t, T)\right|\left\|F^{2}(\sqrt{v}-\Phi(t, v(0))) \frac{\partial^{2} \widetilde{V}}{\partial F^{2}}\right\|_{L^{2}(\Omega)} \\
& \leqslant\left|\rho_{F, r} \eta B_{r}(t, T)\right| F_{\max }^{2}|\sqrt{v}-\Phi(t, v(0))|\left\|\frac{\partial^{2} \widetilde{V}}{\partial F^{2}}\right\|_{L^{2}(\Omega)} .
\end{aligned}
$$

Then, we have

$$
\|e\|_{L^{2}(\Omega)} \leqslant \frac{1}{\alpha} \sup _{s \in(0, t)}\left(\left|\rho_{F, r} \eta B_{r}(t, T)\right| F_{\max }^{2}|\sqrt{v}-\Phi(t, v(0))|\left\|\frac{\partial^{2} \widetilde{V}}{\partial F^{2}}\right\|_{L^{2}(\Omega)}\right) .
$$


This latter inequality bound gives an accurate representation of the parameters and functions that have an impact on the error made when solving for $\widetilde{V}$, as an approximation for $V$. The errors between $V$ and $\widetilde{V}$ can be bounded by the above inequality for large values of $v$, which is illustrated in Fig. 2 in Section 5 . This is because the values of $\frac{\partial^{2} \widetilde{V}}{\partial F^{2}}$ are very small for large $v$-values, which is also presented in Fig. 2.

\section{Numerical validation of the error analysis}

In this section, we will solve numerically, by means of the ADI finite difference method, the HHW PDE (2.11), its approximating PDE (2.12) and the PDE for the error $e$, to confirm the error analysis from the previous section.

\subsection{ADI finite difference method and grid stretching}

The discretization and solution techniques follow very closely the detailed description of ADI in [12].

PDE (2.11) and its approximating PDE (2.12) are semi-discretized on a nonuniform Cartesian spatial grid, as described below (see, however, also [12]).

In the $F$-direction, we define a nonuniform mesh by placing relatively many grid points in a prescribed interval $\left[F_{\text {left }}, F_{\text {right }}\right] \subseteq\left[0, F_{\text {max }}\right]$, which contains the strike price, $K$. In the interval $\left[F_{\text {left }}, F_{\text {right }}\right]$, the parameters $\xi_{\min }=\xi_{0}<\xi_{1}<$ $\cdots<\xi_{M}=\xi_{\max }$ are given by

$$
\xi_{\text {min }}=\operatorname{arcsinh}\left(-\frac{F_{\text {left }}}{d_{1}}\right), \quad \xi_{\max }=\xi_{\text {int }}+\operatorname{arcsinh}\left(\frac{F_{\text {max }}-F_{\text {right }}}{d_{1}}\right), \quad \xi_{\text {int }}=\frac{F_{\text {right }}-F_{\text {left }}}{d_{1}},
$$

where integer $M \geqslant 1$ and parameter $d_{1}>0$. It is clear that $\xi_{\min }<0<\xi_{\text {int }}<\xi_{\max }$.

Then, the grid points $0=F_{0}<F_{1}<\cdots<F_{M}=F_{\max }$ are defined via the following transformation

$$
F_{i}= \begin{cases}F_{\text {left }}+d_{1} \sinh \left(\xi_{i}\right), & \xi_{\text {min }} \leqslant \xi_{i}<0, \\ F_{\text {left }}+d_{1} \xi_{i}, & 0 \leqslant \xi_{i} \leqslant \xi_{\text {int }}, \\ F_{\text {right }}+d_{1} \sinh \left(\xi_{i}-\xi_{\text {int }}\right), & \xi_{\text {int }}<\xi_{i} \leqslant \xi_{\text {max }} .\end{cases}
$$

Parameter $d_{1}$ governs the fraction of points that lie in the interval [ $\left.F_{\text {left }}, F_{\text {right }}\right]$. It is natural to place relatively many mesh grids in this interval, as $F=K$ is of practical relevance, and it alleviates numerical difficulties due to the discontinuous derivative of the payoff function at $F=K$. The $F_{i}$-grid points are uniform in $\left[F_{\text {left }}, F_{\text {right }}\right]$ and nonuniform at other places.

In the $v$-direction, we also define a nonuniform mesh, as in [14]. With integer $N \geqslant 1$ and parameter $d_{2}>0$, the parameters $\eta_{0}<\eta_{1}<\cdots<\eta_{N}$ are then given by $\eta_{j}=j \cdot \Delta \eta(0 \leqslant j \leqslant N), \Delta \eta=\frac{1}{N} \operatorname{arcsinh}\left(\frac{v_{\max }}{d_{2}}\right)$, and $0=v_{0}<v_{1}<\cdots<v_{N}<v_{\max }$ are defined by

$$
v_{j}=d_{2} \sinh \left(\eta_{j}\right), \quad 0 \leqslant j \leqslant N .
$$

Parameter $d_{2}$ controls the number of grid points $v_{j}$ in the vicinity of $v=0$. We should place relatively many grid points near $v=0$, because PDE (2.11) and its approximation (2.12) become convection-dominated PDEs in the $v$-direction, when $v \rightarrow 0$.

The finite difference discretization employed on the stretched grid is relatively standard. Let $V: \mathbb{R} \rightarrow \mathbb{R}$ be a function on a mesh $\left\{x_{i}, y_{j}\right\}, i=0,1, \ldots, M, j=0,1, \ldots, N$, with mesh widths $\Delta x_{i}=x_{i}-x_{i-1}, \Delta y_{j}=y_{j}-y_{j-1}$. We employ the following well-known finite difference schemes to approximate the first and second derivatives of $V$ at the point $\left(x_{i}, y_{j}\right)$ :

$$
\begin{aligned}
& \frac{\partial V\left(t, x_{i}, y_{j}\right)}{\partial x} \approx \alpha_{-2} V\left(t, x_{i-2}, y_{j}\right)+\alpha_{-1} V\left(t, x_{i-1}, y_{j}\right)+\alpha_{0} V\left(t, x_{i}, y_{j}\right), \\
& \frac{\partial V\left(t, x_{i}, y_{j}\right)}{\partial x} \approx \beta_{-1} V\left(t, x_{i-1}, y_{j}\right)+\beta_{0} V\left(t, x_{i}, y_{j}\right)+\beta_{1} V\left(t, x_{i+1}, y_{j}\right), \\
& \frac{\partial V\left(t, x_{i}, y_{j}\right)}{\partial x} \approx \gamma_{0} V\left(t, x_{i}, y_{j}\right)+\gamma_{1} V\left(t, x_{i+1}, y_{j}\right)+\gamma_{2} V\left(t, x_{i+2}, y_{j}\right), \\
& \frac{\partial^{2} V\left(t, x_{i}, y_{j}\right)}{\partial x^{2}} \approx \delta_{-1} V\left(t, x_{i-1}, y_{j}\right)+\delta_{0} V\left(t, x_{i}, y_{j}\right)+\delta_{1} V\left(t, x_{i+1}, y_{j}\right),
\end{aligned}
$$

where

$$
\begin{aligned}
& \alpha_{-2}=\frac{\Delta x_{i}}{\Delta x_{i-1}\left(\Delta x_{i-1}+\Delta x_{i}\right)}, \quad \alpha_{-1}=\frac{-\Delta x_{i-1}-\Delta x_{i}}{\Delta x_{i-1} \Delta x_{i}}, \quad \alpha_{0}=\frac{\Delta x_{i-1}+2 \Delta x_{i}}{\Delta x_{i}\left(\Delta x_{i-1}+\Delta x_{i}\right)}, \\
& \beta_{-1}=\frac{-\Delta x_{i+1}}{\Delta x_{i}\left(\Delta x_{i}+\Delta x_{i+1}\right)}, \quad \beta_{0}=\frac{\Delta x_{i+1}-\Delta x_{i}}{\Delta x_{i} \Delta x_{i+1}}, \quad \beta_{1}=\frac{\Delta x_{i}}{\Delta x_{i+1}\left(\Delta x_{i}+\Delta x_{i+1}\right)}, \\
& \gamma_{0}=\frac{-2 \Delta x_{i+1}-\Delta x_{i+2}}{\Delta x_{i+1}\left(\Delta x_{i+1}+\Delta x_{i+2}\right)}, \quad \gamma_{1}=\frac{\Delta x_{i+1}+\Delta x_{i+2}}{\Delta x_{i+1} \Delta x_{i+2}}, \quad \gamma 2=\frac{-\Delta x_{i+1}}{\Delta x_{i+2}\left(\Delta x_{i+1}+\Delta x_{i+2}\right)},
\end{aligned}
$$




$$
\delta_{-1}=\frac{2}{\Delta x_{i}\left(\Delta x_{i}+\Delta x_{i+1}\right)}, \quad \delta_{0}=\frac{-2}{\Delta x_{i} \Delta x_{i+1}}, \quad \delta_{1}=\frac{2}{\Delta x_{i+1}\left(\Delta x_{i}+\Delta x_{i+1}\right)} .
$$

Here, Eqs. (4.4) and (4.6) are the central schemes, whereas Eqs. (4.3) and (4.5) represent the backward and forward schemes, respectively. The mixed derivative, $\frac{\partial^{2} V\left(t, x_{i}, y_{j}\right)}{\partial x \partial y}$, can be approximated by successive application of the scheme (4.4) in $x$ - and $y$-directions, resulting in a nine-point discretization.

Remark 2. The PDE discretization for the approximation $\widetilde{V}$ will only differ with respect to the square-root term, i.e. in the discretization for $\widetilde{V}$ we will have

$$
\sqrt{v_{j}} \rightarrow \Phi(n \Delta t, v(0)), \quad j=0, \ldots N, n=0, \ldots, L,
$$

where function $\Phi(n \Delta t, v(0))$ is defined in (2.13), $v(0)$ is the initial variance and $\Delta t=T / L$. In the sub-domain $v>\bar{v}$, backward scheme (4.3) is applied for $\frac{\partial \widetilde{V}}{\partial v}$, which alleviates spurious oscillations in the finite difference solution when $\gamma$ is small.

\subsection{Details of boundary and final conditions}

In the numerical experiments to follow, we will consider a European put option, for which we get the following set of final and boundary conditions:

$$
V(T, F(T), v(T))=\max (K-F(T), 0),
$$

and

$$
\left.V\right|_{F=0}=K,\left.\quad V\right|_{F=F_{\max }}=0,\left.\quad \frac{\partial V}{\partial v}\right|_{v=v_{\max }}=0
$$

whereas for $v=0$ we employ:

$$
\kappa \bar{v} \frac{\partial V}{\partial v}+\frac{\partial V}{\partial t}+\frac{1}{2} \eta^{2} B_{r}^{2}(t, T) F^{2} \frac{\partial^{2} V}{\partial F^{2}}=0 .
$$

This is the equation resulting when $v$ tends to zero in the PDE. Here, the term $\frac{\partial V}{\partial v}$ is approximated by the forward scheme (4.5) [12], and for the time discretization we will consider the alternating direction implicit (ADI) method in Section 4.4.

With $\Delta F_{i}=F_{i+1}-F_{i}(i=0, \ldots, M-1), \Delta F_{M}=\Delta F_{M-1}, \Delta v_{j}=v_{j+1}-v_{j}(j=0, \ldots, N-1), \Delta v_{N}=\Delta v_{N-1}$, these final and boundary conditions give us the following discretization:

$$
\begin{aligned}
& V_{i, j}^{L}=\max \left(K-i \Delta F_{i}, 0\right), \quad i=0, \ldots, M, j=0, \ldots, N, \\
& V_{0, j}^{n}=K, \quad j=0, \ldots, N, n=0, \ldots, L-1, \\
& V_{M, j}^{n}=0, \quad j=0, \ldots, N, n=0, \ldots, L-1, \\
& V_{i, N}^{n}=V_{i, N-1}^{n}, \quad i=0, \ldots, M, n=0, \ldots, L-1 .
\end{aligned}
$$

\subsection{The PDE for the error}

By combining the full-scale HHW option pricing PDE (2.11) and its approximating PDE (2.12) and defining $e:=V-\widetilde{V}$, we obtain the following PDE for error $e$ :

$$
\begin{aligned}
-\frac{\partial e}{\partial t}= & \kappa(\bar{v}-v) \frac{\partial e}{\partial v}+\frac{1}{2} \gamma^{2} v \frac{\partial^{2} e}{\partial v^{2}}+\rho_{F, v} F \gamma v \frac{\partial^{2} e}{\partial F \partial v}+F^{2}\left(\frac{1}{2} v+\frac{1}{2} \eta^{2} B_{r}^{2}(t, T)-\rho_{F, r} B_{r}(t, T) \eta \sqrt{v}\right) \frac{\partial^{2} e}{\partial F^{2}} \\
& +F^{2} \rho_{F, r} \eta B_{r}(t, T)(\Phi(t, v(0))-\sqrt{v}) \frac{\partial^{2} \widetilde{V}}{\partial F^{2}} .
\end{aligned}
$$

The final and boundary conditions of the error PDE (4.8) are given by $e(T, F(T), v(T))=0$, and,

$$
\left.e\right|_{F=0}=0,\left.\quad e\right|_{F=F_{\max }}=0,\left.\quad \frac{\partial e}{\partial v}\right|_{v=v_{\max }}=0,
$$

and for $v=0$ we employ here:

$$
\kappa \bar{v} \frac{\partial e}{\partial v}+\frac{\partial e}{\partial t}+\frac{1}{2} \eta^{2} B_{r}^{2}(t, T) F^{2} \frac{\partial^{2} e}{\partial F^{2}}=-\rho_{F, r} F^{2} \eta B_{r}(t, T) \Phi(t, v(0)) \frac{\partial^{2} \widetilde{V}}{\partial F^{2}} .
$$


Table 1

Sets of parameters used in the experiments. Parameters for the asset process are defined as follows, Set 1 [20] (Feller condition is satisfied), and Set 2 (Feller condition is not satisfied). Maturity $T$ is chosen to 1 year and strike $K=1$.

\begin{tabular}{llllllll}
\hline Parameter & $\kappa$ & $\bar{v}$ & $\gamma$ & $\rho_{F, v}$ & $\lambda$ & $\rho_{F, r}$ & $\eta$ \\
\hline Set 1 & 2.5 & 0.06 & 0.5 & -0.1 & 1.5 & 0.05 & 0.4 \\
Set 2 & 0.6067 & 0.0707 & 0.5 & -0.7571 & 0.01 & 0.005 & 0.3 \\
\hline
\end{tabular}

Its discretization is essentially the same as that of boundary condition (4.7).

These final and boundary conditions give us the following discretization:

$$
\begin{aligned}
& e_{i, j}^{L}=0, \quad i=0, \ldots, M, j=0, \ldots, N, \\
& e_{0, j}^{n}=0, \quad j=0, \ldots, N, n=0, \ldots, L-1, \\
& e_{M, j}^{n}=0, \quad j=0, \ldots, N, n=0, \ldots, L-1, \\
& e_{i, N}^{n}=e_{i, N-1}^{n}, \quad i=0, \ldots, M, n=0, \ldots, L-1 .
\end{aligned}
$$

\subsection{Time discretization and ADI scheme}

After discretizing PDE (2.11), its approximating PDE (2.12) or the error PDE, we are left with the following final value problem for a system of ordinary differential equations (ODEs):

$$
V_{h}^{\prime}(t)=A_{h}(t) V_{h}(t)+g_{h}(t), \quad V_{h}(T)=V_{T}
$$

Here, $V_{h}(t)$ is an unknown vector composed of the approximations of the option values at the spatial grid points, $V_{h}(T)$ is obtained by the final conditions, $A_{h}(t), 0 \leqslant t \leqslant T$ is a given real square matrix, and $g_{h}(t)$ is a given real vector that is obtained from the boundary conditions.

For the time discretization and solution of (4.9), we consider the splitting scheme of alternating direction implicit type, see also [12]. Matrix $A_{h}(t)$ is decomposed into three matrices, $A_{h}(t)=A_{0}+A_{1}(t)+A_{2}$, where $A_{0}$ represents the finite difference discretization of all mixed derivatives originating from the discrete PDE (2.11), or its approximation (2.12), $A_{1}(t)$ and $A_{2}$ are composed of the finite difference discretizations of the spatial derivatives in the $F$ - and $v$-directions, respectively (both matrices $A_{1}(t)$ and $A_{2}$ are tridiagonal). Vector $g(t)$ is decomposed analogously to $A_{h}(t)$, i.e. $g(t)=g_{0}+g_{1}(t)+g_{2}$.

We will apply the first-order accurate Douglas scheme to solve the ODEs, as follows. With $L \geqslant 1, t_{n}=n \Delta t$ ( $n=L, L-$ $1, \ldots, 1,0), \Delta t=\frac{T}{L}, \Delta g_{n}=g\left(t_{n-1}\right)-g\left(t_{n}\right)$, and $\theta>0$ a given real parameter, the Douglas scheme reads:

$$
\begin{aligned}
& Y_{0}=V_{n}+\Delta t\left(A_{h}\left(t_{n}\right) V_{n}+g\left(t_{n}\right)\right), \\
& Y_{1}=Y_{0}+\theta \Delta t A_{2}\left(Y_{1}-V_{n}\right), \\
& Y_{2}=Y_{1}+\theta \Delta t\left(A_{1}\left(t_{n-1}\right) Y_{2}-A_{1}\left(t_{n}\right) V_{n}+\Delta g_{n}\right), \\
& V_{n-1}=Y_{2},
\end{aligned}
$$

which generates the successive approximations, $V_{n}$, to the solutions $V\left(t_{n}, F_{i}, v_{j}\right)$.

Matrix $A_{0}$ representing the mixed derivatives is treated in an explicit form, see (4.10) in the above scheme. The matrices $A_{1}(t)$ and $A_{2}$, representing the $F$ - and $v$-direction derivatives, respectively, are treated in an implicit form as expressed in (4.11) and (4.12). Because these latter steps generate tridiagonal matrices, we can solve these systems by a basic $L U$ factorization in optimal complexity.

\section{Numerical results}

In this section, we present some numerical tests with the ADI scheme to solve PDE (2.11), its approximating PDE (2.12) and the error PDE (4.8). A comparison between the results obtained by the ADI scheme and COS method is also given.

We perform numerical experiments where different parameter sets are considered. In the experiments, we have chosen a basis domain with $F_{\max }=3, v_{\max }=2$, so that $(F, v) \in[0,3] \times[0,2]$, with $F_{0}=K=1$. We will vary the domain size in one of the numerical experiments.

For the numerical discretization we have used $\theta=\frac{2}{3}, F_{\text {left }}=0.8, F_{\text {right }}=1.2, d_{1}=2, d_{2}=\frac{1}{250}$, see Eq. (4.2), and the sets of SDE parameters given in Table 1. To validate our numerical schemes, we first compare the solution, $\widetilde{V}$, of the approximate HHW PDE (2.12) by the ADI scheme with the numerical solution for the same PDE by means of the COS method. Table 2 presents this error in two different norms, for the four test cases, where the Feller condition is satisfied, and where the Feller condition is not satisfied. For the results in the table, the max-error is defined as

$$
\max _{i}\left|\widetilde{V}_{\cos }\left(t_{0}, F_{i}, v_{0}\right)-\widetilde{V}_{\mathrm{ADI}}\left(t_{0}, F_{i}, v_{0}\right)\right|
$$


Table 2

The errors between the $\mathrm{ADI}$ and $\operatorname{COS}$ methods, at $v=v_{0}$, when the Feller condition is satisfied (Results 1) and not satisfied (Results 2), with parameters in Set 1 and Set 2 in Table 1, respectively.

\begin{tabular}{llllr}
\hline Result & $\#$ grid points of $\mathrm{F} / \mathrm{v} / \mathrm{t}$ & $L_{2}$-error & max-error & point $\left(F_{i}\right)$ of max-error \\
\hline 1 & $30 / 30 / 10$ & 0.0035 & 0.0049 & 1.0333 \\
& $60 / 60 / 20$ & 0.0017 & 0.0024 & 1.0333 \\
2 & $30 / 30 / 10$ & 0.0050 & 0.0050 & 1.1730 \\
& $60 / 60 / 20$ & 0.0025 & 0.0024 & 1.1498 \\
\hline
\end{tabular}

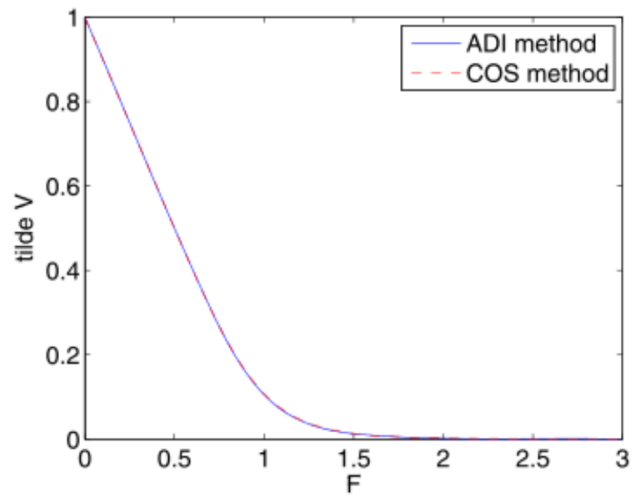

(a)

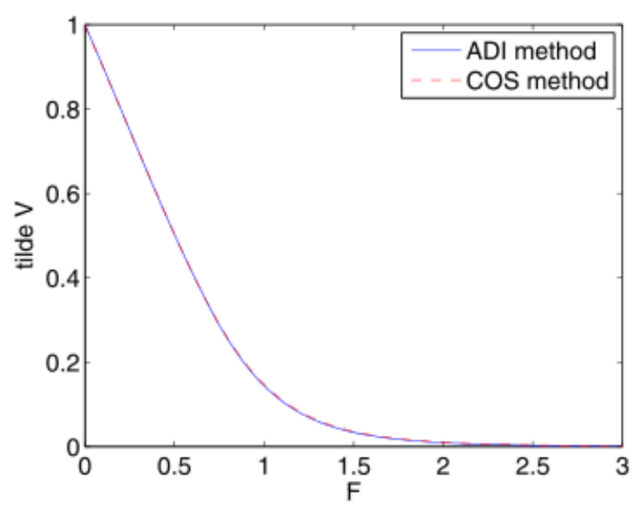

(c)

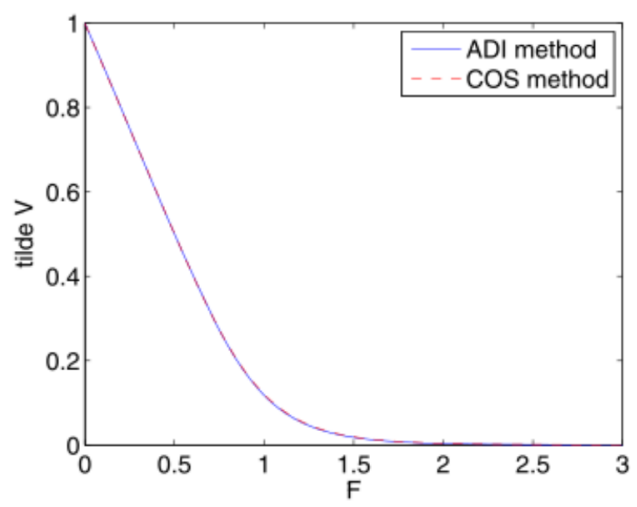

(e)

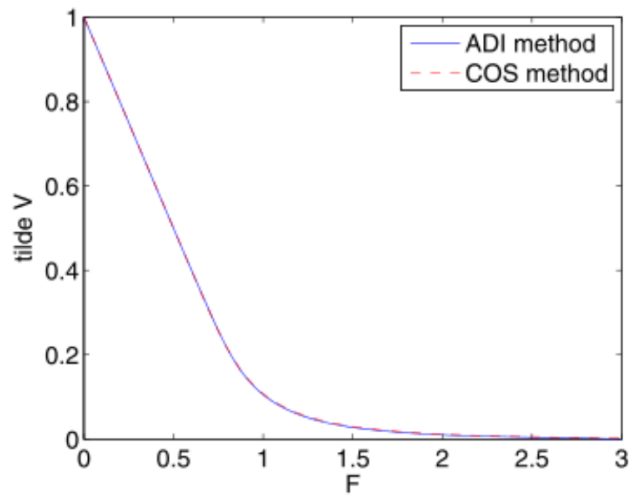

(b)

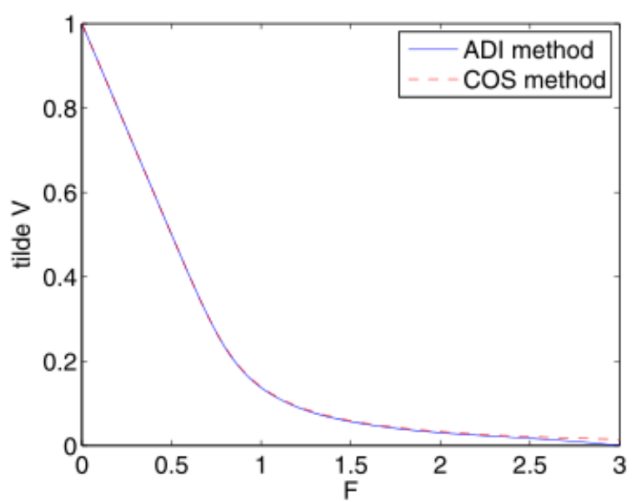

(d)

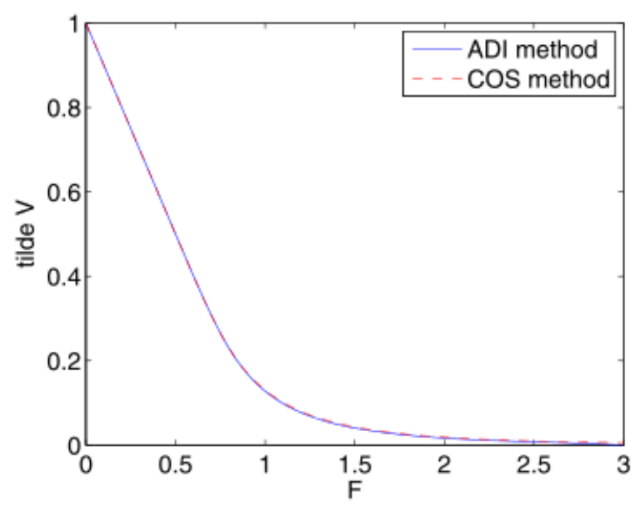

(f)

Fig. 1. Comparison between the ADI and COS method for $\widetilde{V}$. Plot (a): Test case 1 (Feller condition is satisfied), $T=1$; Plot (b): Test case 2 (Feller condition is not satisfied), $T=1$; Plot (c): Test case $1, T=2$; Plot (d): Test case $2, T=2$. Plot (e): Test case 1 with $v_{0}=0.15, T=1$. Plot (f): Test case 2 with $v_{0}=0.15$, $T=1$. The parameters are in Table 1. 


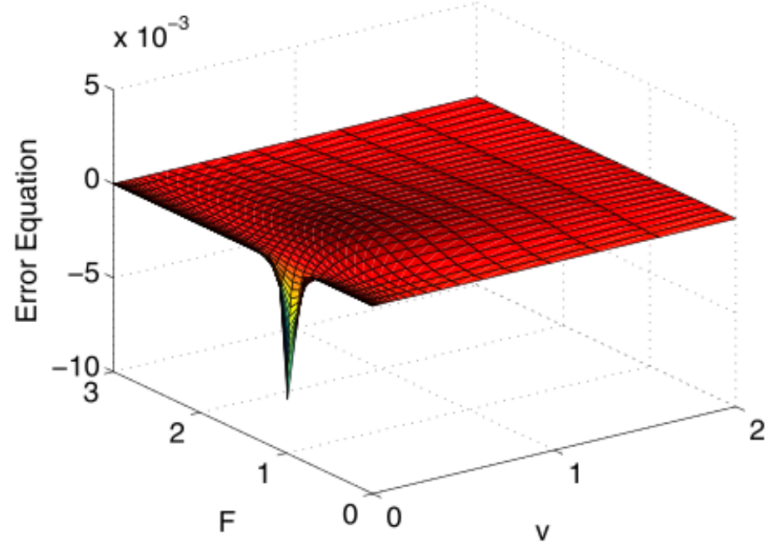

(a)

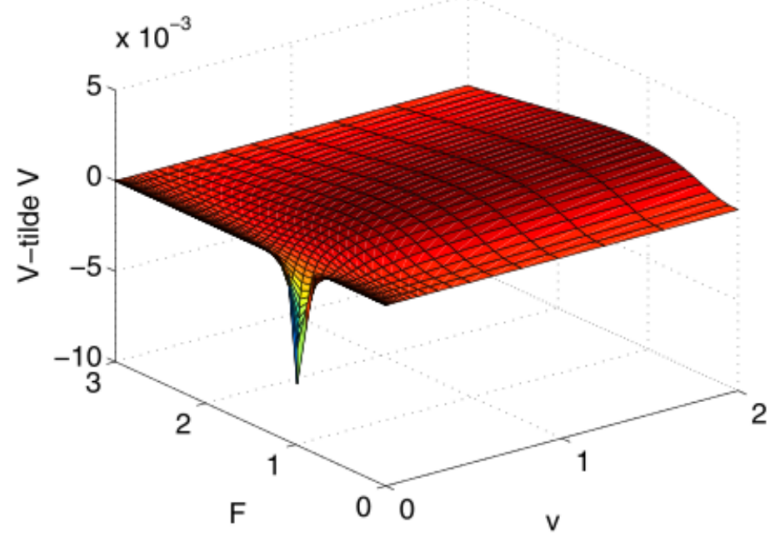

(c)

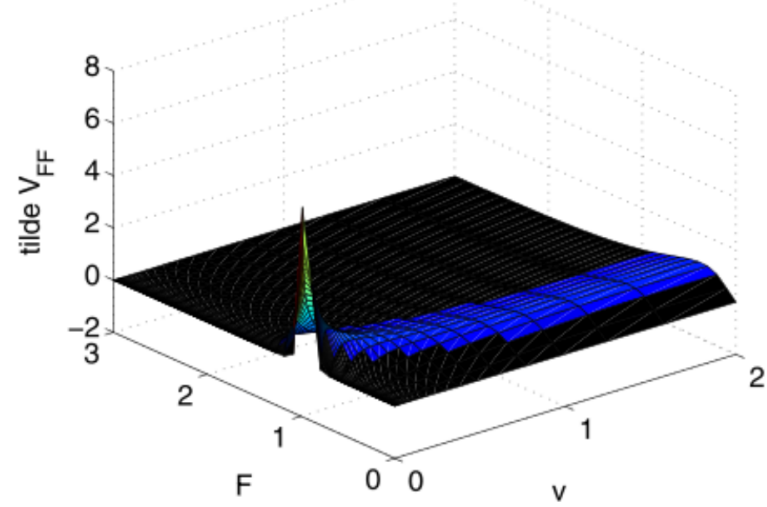

(e)

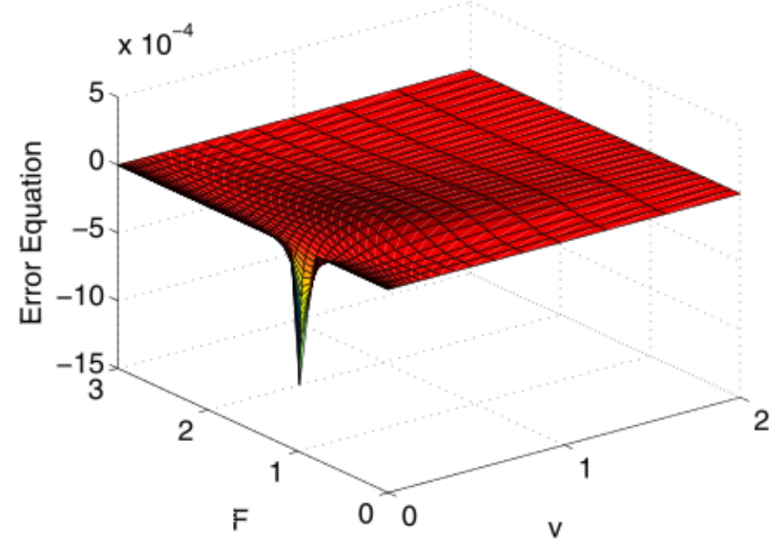

(b)

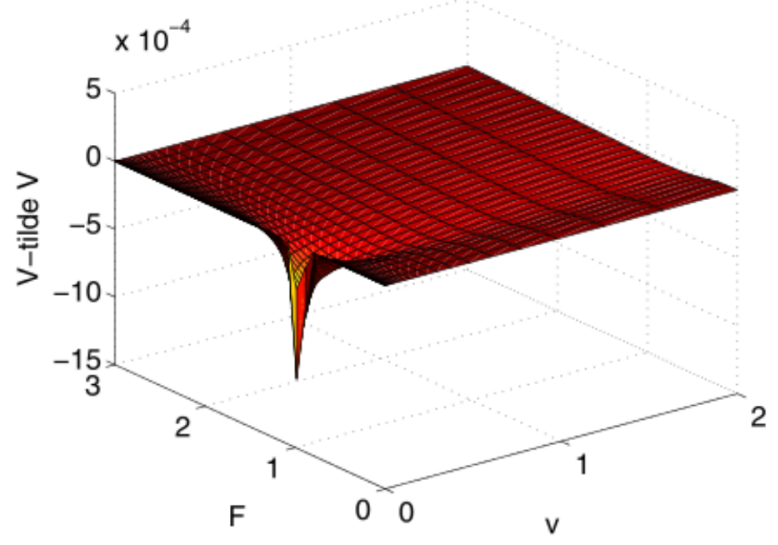

(d)

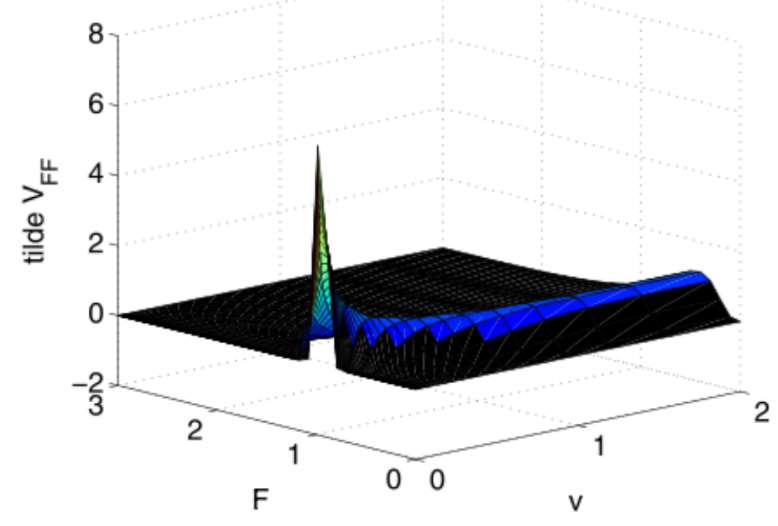

(f)

Fig. 2. Comparison of solutions from the full-scale and approximate HHW PDE; (a): values of error PDE via ADI scheme (Set 1); (b): values of error PDE via ADI scheme (Set 2); (c): values of $V-\widetilde{V}$ (Set 1); (d): values of $V-\widetilde{V}$ (Set 2); (e): values of $\widetilde{V}_{\mathrm{FF}}$ (Set 1); (f): values of $\widetilde{V}_{\mathrm{FF}}($ Set 2 ). The parameters are in Table 1. 
Table 3

The effect of parameter variations for $\rho_{F, r}, \eta, \lambda, F_{\max }$ and $v_{0}$ on the $\|V-\widetilde{V}\|$ via Approximation I with parameters in Set 1 in Table 1.

\begin{tabular}{|c|c|c|c|c|}
\hline 1 & $\begin{array}{l}\rho_{F, r} \\
\|V-\widetilde{V}\|\end{array}$ & $\begin{array}{l}0.4 \\
5.4759 \times 10^{-4}\end{array}$ & $\begin{array}{l}0.04 \\
5.5650 \times 10^{-5}\end{array}$ & $\begin{array}{l}0.004 \\
5.5746 \times 10^{-6}\end{array}$ \\
\hline 2 & $\begin{array}{l}\eta \\
\|V-\tilde{V}\|\end{array}$ & $\begin{array}{l}0.5 \\
0.0045\end{array}$ & $\begin{array}{l}0.05 \\
5.4759 \times 10^{-4}\end{array}$ & $\begin{array}{l}0.005 \\
5.5770 \times 10^{-5}\end{array}$ \\
\hline 3 & $\stackrel{\lambda}{\|V-\tilde{V}\|}$ & $\begin{array}{l}1.5 \\
5.4759 \times 10^{-4}\end{array}$ & $\begin{array}{l}0.15 \\
0.0058\end{array}$ & $\begin{array}{l}0.015 \\
0.0060\end{array}$ \\
\hline 4 & $\begin{array}{l}F_{\max } \\
\|V-\tilde{V}\|\end{array}$ & $\begin{array}{l}3 \\
5.4759 \times 10^{-4}\end{array}$ & $\begin{array}{l}4 \\
5.4159 \times 10^{-4}\end{array}$ & $\begin{array}{l}11 \\
5.0480 \times 10^{-4}\end{array}$ \\
\hline 5 & $\begin{array}{l}v_{0} \\
\|V-\tilde{V}\|\end{array}$ & $\begin{array}{l}0.1 \\
5.4759 \times 10^{-4}\end{array}$ & $\begin{array}{l}0.01 \\
6.2149 \times 10^{-4}\end{array}$ & $\begin{array}{l}0.001 \\
7.0032 \times 10^{-4}\end{array}$ \\
\hline
\end{tabular}

and the $L_{2}$-error is defined as

$$
\left[\sum_{i=0}^{M} \Delta F_{i}\left(\widetilde{V}_{\cos }\left(t_{0}, F_{i}, v_{0}\right)-\widetilde{V}_{\mathrm{ADI}}\left(t_{0}, F_{i}, v_{0}\right)\right)^{2}\right]^{\frac{1}{2}},
$$

because we can use the COS method in a straightforward way to compute the option values for one variance value, $v_{0}=v(0)$ only.

The maximum errors in Table 2 are sufficiently small, compared to the option values at the grid points for which the maximum is achieved, so that we can consider our numerical implementation to be correct (after several of these checks). The convergence of the PDE solution to the COS solution is also observed in Table 2. Doubling the number of grid points reduces the ( $L_{2}$ - and max-) errors by a factor two.

Fig. 1 presents the difference between the two $\widetilde{V}$-solutions, for a fixed value of $v$ and two different maturities $T$ ( $T=1$ and $T=2$ ) graphically. From this figure, we can see that the approximation is also accurate for a longer maturity and other values of $v_{0}$.

Remark 3. The cost of the ADI time-stepping FD method increases with the number of time-steps, because it is based on a discretization in time. When dealing with European options, time-stepping is not needed in the discounted expected payoff approach. This appears an advantage in terms of CPU time of Fourier techniques whenever they are applicable. PDE techniques are however advantageous in terms of their general applicability (as there is no need for approximations).

As a next step, to confirm the error analysis in Section 3.2, with $\Phi(t, v(0))$ the approximation in Eqs. (2.13) and (2.14)

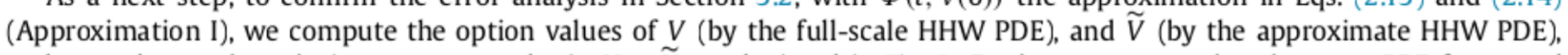
and we subtract the solution vectors to obtain $V-\widetilde{V}$, as depicted in Fig. 2. Furthermore, we solve the error PDE for $e$, and we also display the second derivative $\widetilde{V}_{\mathrm{FF}}$ which plays a prominent role in the error analysis. The values of $\widetilde{V}_{\mathrm{FF}}$ are computed via the finite difference method. Fig. 2 shows that for both cases of the Feller condition satisfied and not satisfied in Table 1 , the solution, $\widetilde{V}$, originating from the approximate HHW PDE agrees very well with the solution $V$, of the full-scale HHW PDE. The results for $t=0$ are displayed. In Fig. 2 we observe that both quantities, $V-\widetilde{V}$ as well as $e$, exhibit an error peak at the position $F=K, v=0$. This peak corresponds to the peak appearing in the second derivative $\widetilde{V}_{\mathrm{FF}}$, which is in agreement with the error analysis in Section 3.2. The size of this peak is however very moderate. In addition, we can see from plots (a) and (b) in Fig. 2 that $e$ is very small at $v=v_{\max }$ compared to the values at $v=0$, which means that the error (3.25) can also be bounded for a very large $v$. In particular, the values of $\widetilde{V}_{\mathrm{FF}}$ are very small, which is depicted in plots (e) and (f).

We then check quantitatively the influence of the parameters $\rho_{F, r}, \eta, \lambda, F_{\max }$ and $v_{0}$ on the error $V-\widetilde{V}$. We do this by varying each parameter value from Table 1 . The results for the error are displayed in Tables 3 and 4 for Set 1 and Set 2 , respectively. The norm $\|V-\widetilde{V}\|$ in these two tables is defined as

$$
\sqrt{\frac{1}{(M+1)(N+1)} \sum_{i=0}^{M} \sum_{j=0}^{N}\left[V\left(t_{0}, F_{i}, v_{j}\right)-\widetilde{V}\left(t_{0}, F_{i}, v_{j}\right)\right]^{2}},
$$

and the numbers of grid points chosen for this experiment are $M=N=30, L=10$.

From Table 3 (Feller condition satisfied), we can see that the parameters $\rho_{F, r}$ and $\eta$ have a pronounced, linear effect on the values of $\|V-\widetilde{V}\|$. This is in agreement with the error analysis bound in (3.24). Other parameters do not affect the error $\|V-\widetilde{V}\|$ significantly. However, we can see that, when $\lambda$ decreases, the errors become slightly bigger. When $F_{\max }$ increases, the errors become slightly smaller.

The results in Table 4 (Feller condition not satisfied) also show that decreasing the values of $\rho_{F, r}, \eta$ and $v_{0}$ leads to a decrease of $\|V-\widetilde{V}\|$, whereas the parameters $\lambda$ and $F_{\max }$ have the opposite effects on $\|V-\widetilde{V}\|$. These two tables serve 
Table 4

The effect of parameter variations for $\rho_{F, \mathrm{r}}, \eta, \lambda, F_{\max }$ and $v_{0}$ on the $\|V-\widetilde{V}\|$ via Approximation I with parameters in Set 2 in Table 1.

\begin{tabular}{|c|c|c|c|c|}
\hline 1 & $\begin{array}{l}\rho_{F, r} \\
\|V-\widetilde{V}\|\end{array}$ & $\begin{array}{l}0.3 \\
1.4750 \times 10^{-4}\end{array}$ & $\begin{array}{l}0.03 \\
1.4904 \times 10^{-5}\end{array}$ & $\begin{array}{l}0.003 \\
1.4920 \times 10^{-6}\end{array}$ \\
\hline 2 & $\eta_{\|V-\widetilde{V}\|}$ & $\begin{array}{l}0.5 \\
0.0082\end{array}$ & $\begin{array}{l}0.05 \\
0.0013\end{array}$ & $\begin{array}{l}0.005 \\
1.4750 \times 10^{-4}\end{array}$ \\
\hline 3 & $\stackrel{\lambda}{\|V-\widetilde{V}\|}$ & $\begin{array}{l}0.1 \\
1.4290 \times 10^{-4}\end{array}$ & $\begin{array}{l}0.01 \\
1.4750 \times 10^{-4}\end{array}$ & $\begin{array}{l}0.001 \\
1.4797 \times 10^{-4}\end{array}$ \\
\hline 4 & $\begin{array}{l}F_{\max } \\
\|V-\widetilde{V}\|\end{array}$ & $\frac{3}{1.4750 \times 10^{-4}}$ & $\begin{array}{l}4 \\
1.4408 \times 10^{-4}\end{array}$ & $\begin{array}{l}11 \\
1.3467 \times 10^{-4}\end{array}$ \\
\hline 5 & $\begin{array}{l}v_{0} \\
\|V-\widetilde{V}\|\end{array}$ & $\begin{array}{l}0.1 \\
1.4750 \times 10^{-4}\end{array}$ & $\begin{array}{l}0.01 \\
1.0608 \times 10^{-5}\end{array}$ & $\begin{array}{l}0.001 \\
1.1571 \times 10^{-4}\end{array}$ \\
\hline
\end{tabular}

\section{Table 5}

The effect of the parameters $\kappa, \bar{v}, \gamma$, and $v_{0}$ on the $\max \left|\mathbb{E}_{1}-\mathbb{E}_{I I}\right|$. The parameters are $\rho_{F, v}=-0.3, \lambda=1.1, \eta=0.05, \rho_{F, r}=0.3,(1): \bar{v}=0.6, \gamma=0.3$, $v_{0}=0.1 ;(2), \kappa=1.5, \gamma=0.4, v_{0}=0.2 ;(3), \kappa=2.1, \bar{v}=0.6, v_{0}=0.3 ;(4), \kappa=2.5, \bar{v}=0.5, \gamma=0.6$.

\begin{tabular}{|c|c|c|c|c|}
\hline \multirow[t]{3}{*}{1} & $\kappa$ & 0.02 & 0.2 & 2 \\
\hline & $\max \left|\mathbb{E}_{1}-\mathbb{E}_{\| I}\right|$ & 0.0026 & 0.0012 & 0.0224 \\
\hline & $\max \left|\widetilde{V}_{1}-\widetilde{V}_{11}\right|$ & $1.8389 \times 10^{-5}$ & $1.9593 \times 10^{-5}$ & $3.6407 \times 0^{-4}$ \\
\hline \multirow[t]{3}{*}{2} & $\bar{v}$ & 0.005 & 0.05 & 0.5 \\
\hline & $\max \left|\mathbb{E}_{1}-\mathbb{E}_{\| I}\right|$ & 0.0129 & 0.0056 & 0.0045 \\
\hline & $\max \left|\widetilde{V}_{I}-\widetilde{V}_{I I}\right|$ & $1.3312 \times 10^{-4}$ & $9.4293 \times 10^{-5}$ & $7.2739 \times 10^{-5}$ \\
\hline \multirow[t]{3}{*}{3} & $\gamma$ & 0.3 & 0.9 & 1.5 \\
\hline & $\max \left|\mathbb{E}_{1}-\mathbb{E}_{\mathrm{II}}\right|$ & 0.0044 & $9.49695 \times 10^{-4}$ & 0.0116 \\
\hline & $\max \left|\widetilde{V}_{1}-\widetilde{V}_{1 I}\right|$ & $7.0735 \times 10^{-5}$ & $5.2387 \times 10^{-6}$ & $1.2100 \times 10^{-4}$ \\
\hline \multirow[t]{3}{*}{4} & $v_{0}$ & 0.006 & 0.06 & 0.6 \\
\hline & $\max \left|\mathbb{E}_{I}-\mathbb{E}_{I I}\right|$ & 0.0532 & 0.0243 & $8.4550 \times 10^{-4}$ \\
\hline & $\max \left|\widetilde{V}_{1}-\widetilde{V}_{\mathrm{II}}\right|$ & 0.0039 & $3.6451 \times 10^{-4}$ & $1.2388 \times 10^{-5}$ \\
\hline
\end{tabular}

as a numerical confirmation of the error analysis leading to (3.24). Highest errors values are observed for large values of parameter $\eta$. It should be noted, however, that $\eta$-values larger than 0.1 are exceptional in financial applications.

As a next step we will compare the accuracy of the two approximations presented for $\Phi(t, v(0))$ in Section 2.1, i.e. Approximation I (2.13), and Approximation II (2.15). Fig. 3 shows the expectations obtained by Approximation I and Approximation II and the corresponding values of the difference $\widetilde{V}_{1}-\widetilde{V}_{\text {II }}$ (recall that $t=0$ is a singular point for Approximation I) for the parameters in Sets 1 and 2.

It is observed that for both cases of the Feller condition satisfied and not satisfied, the errors between $\widetilde{V}_{\mathrm{I}}$ and $\widetilde{V}_{\mathrm{II}}$ are very small. We have further seen that when the errors between Approximation I and Approximation II get smaller, then the errors between $\widetilde{V}_{1}$ and $\widetilde{V}_{I I}$ also decrease.

Parameter values are now varied and Table 5 shows that, in the numerical tests performed, from a certain $\kappa$-value the value of $\max \left|\mathbb{E}_{I}-\mathbb{E}_{I I}\right|$ increases when $\kappa$ increases (while for some $\kappa$-values the error decreases). We see a similar effect of parameter $\gamma$. The values of $\max \left|\mathbb{E}_{l}-\mathbb{E}_{I I}\right|$ decrease when $\bar{v}$ increases, and parameter $v_{0}$ has a similar effect.

\section{Conclusion}

In this paper, we studied for European plain vanilla options the difference between the HHW PDE and an approximating PDE. Classical PDE error analysis theory was applied to analyze the quality of the approximation and an error PDE was obtained. The effect of financial parameters $\rho_{F, r}, \eta, \lambda, F_{\max }$ and $v_{0}$ on the errors in the solutions has been determined in this analysis. Numerical experiments confirmed that the error between the solutions originating for the full-scale HestonHull-White PDE and its approximation PDE are reasonably small. The numerical results showed that $\rho_{F, r}$ and $\eta$ have an effect on the error, whereas other parameters did not show any significant effect. The error in the option values obtained was however not exceeding the size $10^{-4}$. In addition, we compared two different approximations for the expectation and its effect on the values of the option value from the approximate HHW PDE.

\section{Acknowledgements}

The authors would like to thank Dr. F.J. Vermolen for helpful discussions and the referees for their valuable suggestions which lead to an improved version. S. Guo would like to thank China Scholarship Council that has provided a scholarship for his research work in The Netherlands. 


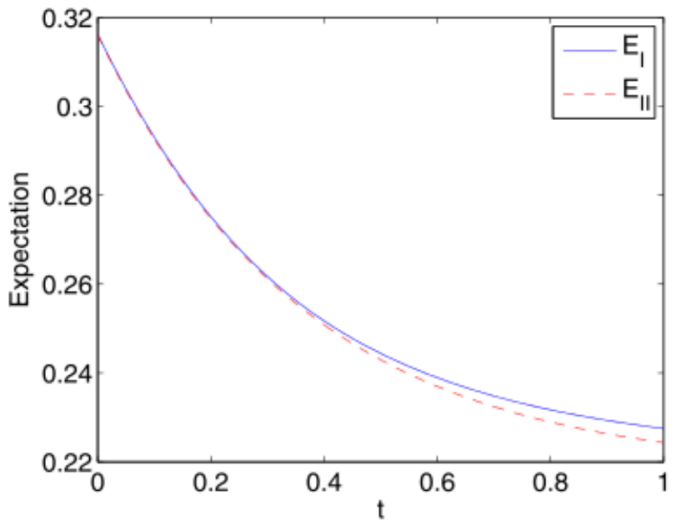

(a)

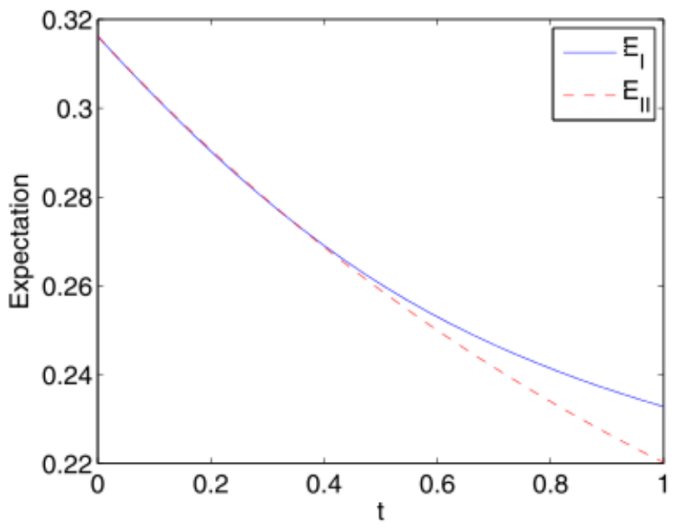

(c)

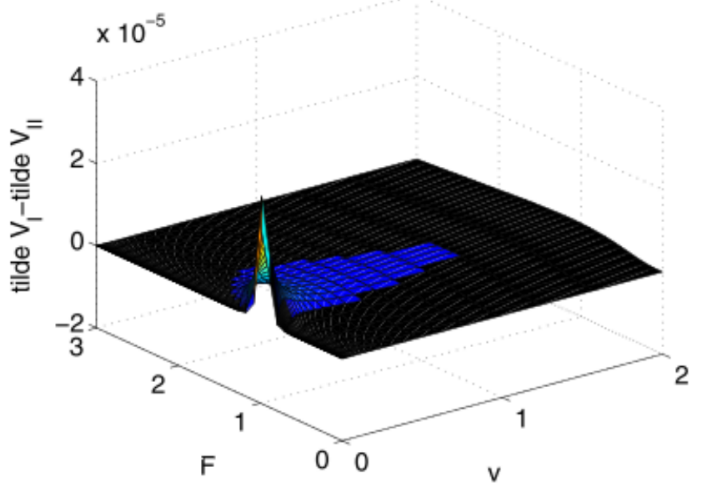

(b)

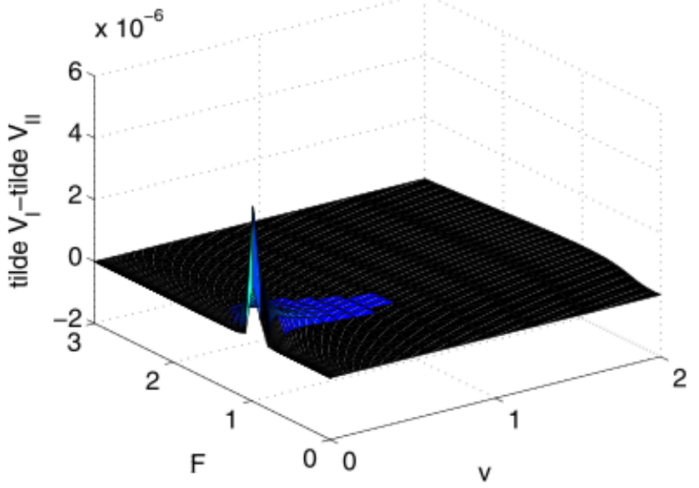

(d)

Fig. 3. Plots (a) and (c): The expectations obtained by Approximation I and II with varying time $t$; (b) and (d): the corresponding option errors $\widetilde{V}_{\mathrm{I}}-\widetilde{V}_{\mathrm{II}}$. The parameters are (a) and (b), Set 1; (c) and (d), Set 2.

\section{References}

[1] Y. Achdou, O. Pironneau, Computational Methods for Option Pricing. SIAM, 2005.

[2] D. Duffie, J. Pan, K. Singleton, Transform analysis and asset pricing for affine jump-diffusions, Econometrica 68 (2000) $1343-1376$.

[3] D. Dufresne, The integrated square-root process, Working paper, University of Montreal, 2001.

[4] E. Ekström, J. Tysk, Boundary conditions for the single-factor term structure equation, Ann. Appl. Probab. 21 (2011) 332-350.

[5] L.C. Evans, Partial Differential Equations, American Mathematical Society, 1997.

[6] F. Fang, C.W. Oosterlee, A novel pricing method for European options based on Fourier-cosine series expansions, SIAM J. Sci. Comput. 31 (2008) $826-848$.

[7] J.-P. Fouque, G. Papanicolaou, K.R. Sircar, Derivatives in Financial Markets With Stochastic Volatility, Cambridge Univ. Press, 2000.

[8] D. Gilbarg, N.S. Trudinger, Elliptic Partial Differential Equations of Second Order, Springer-Verlag, 2000.

[9] L.A. Grzelak, CW. Oosterlee, S. van Weeren, Extension of stochastic volatility models with Hull-White interest rate process, Quant. Finance 12 (1) (2012) 89-105.

[10] L.A. Grzelak, C.W. Oosterlee, On the Heston model with stochastic interest rates, SIAM J. Financ. Math. 2 (2011) $255-285$.

[11] L.A. Grzelak, C.W. Oosterlee, S. van Weeren, The affine Heston model with correlated Gaussian interest rates for pricing hybrid derivatives, Quant. Finance 11 (11) (2011) 1647-1663.

[12] T. Haentjens, K.J. In 't Hout, ADI finite difference schemes for the Heston-Hull-White PDE, J. Comput. Finance 16 (2012) 83-110.

[13] P.S. Hagan, D. Kumar, A.S. Lesniewski, D.E. Woodward, Managing smile risk, Wilmott Mag. 3 (2002) 84-108

[14] K.J. In 't Hout, S. Foulon, ADI finite difference schemes for option pricing in the Heston model with correlation, Int. J. Numer. Anal. Model. 7 (2010) 303-320.

[15] E.E. Kummer, Über die hypergeometrische Reihe $F(a ; b ; x)$. J. Reine Angew. Math. 15 (1936) $39-83$.

[16] J. Necas, Direct Methods in the Theory of Elliptic Equations, Springer-Verlag, 2012.

[17] S. Pagliarani, A. Pascucci, C. Riga, Expansion formulae for local Lévy models, Available at SSRN, http://ssrn.com/abstract=1937149, 2011

[18] A. Pascucci, PDE and Martingale Methods in Option Pricing, Springer-Verlag, 2011.

[19] S. Shreve, Stochastic Calculus for Finance II: Continuous-time Models, Springer Verlag, New York, 2004.

[20] G. Winkler, T. Apel, U. Wystup, in: J. Hakala, U. Wystup (Eds.), Valuation of options in Heston's stochastic volatility model using finite element methods, foreign exchange risk, Risk Publications, 2002. 